\title{
A HEA/MG composite breaks the strength-ductility trade-off by using ultrasonic technology
}

\author{
ZHANG Yong $^{1,2 *} \&$ LI RuiXuan ${ }^{1}$ \\ ${ }^{1}$ Beijing Advanced Innovation Center of Materials Genome Engineering, State Key Laboratory for Advanced Metals and Materials, University \\ of Science and Technology Beijing, Beijing 100083, China; \\ ${ }^{2}$ Qinghai Provincial Key Laboratory of New Light Alloys, Qinghai Provincial Engineering Research Center of High Performance Light Metal \\ Alloys and Forming, Qinghai University, Xining 810016, China
}

Received May 26, 2020; accepted June 11, 2020; published online July 17, 2020

Citation: Zhang Y, Li R X. A HEA/MG composite breaks the strength-ductility trade-off by using ultrasonic technology. Sci China Tech Sci, 2020, 63: 21692170, https://doi.org/10.1007/s11431-020-1675-4

Metallic glasses (MGs) have aroused wide concern for their high strength, large elastic-strain limit and excellent wearresistance, while their applications as the structural materials are severely limited by their room-temperature brittleness. The crystalline in MGs have been used as dendrite/MG composite to improve the plasticity, and nowadays the high entropy alloys (HEAs) emerge from the crystalline alloys with more and more attention for their novel design principles and special properties [1,2]. The composites of HEA/ MG are possibly a good choice for fabricating superior alloys. Significantly different from time-consuming traditional synthesis technique, ultrasonic hammer is a lower energy input with shorter treatment time, and has been successfully applied in the punching of MGs [3] and surface modification of HEAs [4].

In the paper entitled "High-entropy alloy and amorphous alloy composites fabricated by ultrasonic vibrations" [5], the authors fabricated an $\mathrm{Al}_{80} \mathrm{Li}_{5} \mathrm{Mg}_{5} \mathrm{Zn}_{5} \mathrm{Cu}_{5}$ (at\%) lightweight HEA and Zr-based MG composite by using the ultrasonic technology. The composite is composed of $5 \mathrm{wt} \%$ ductile HEA and $95 \mathrm{wt} \%$ brittle MG. The amorphous and crystalline structure does not change under the high frequency vibrations. Furthermore, HEA particles are strongly sandwiched in the amorphous matrix, and the bonding quality is good without pores or cracks. Although the addition of HEA is only $5 \mathrm{wt} \%$, the plasticity is greatly improved by more than ten times accompanied by a slight sacrifice in strength. This is due to the hindering effect on the shear band by the HEA phase, so that the shear band does not directly pass through the alloy.

Strength-ductility trade-off is one of the most important issues in materials science, and this is overcome by the proposed composite using ultrasonic hammer only in several seconds. Furthermore, the preparation process and alloy ra-
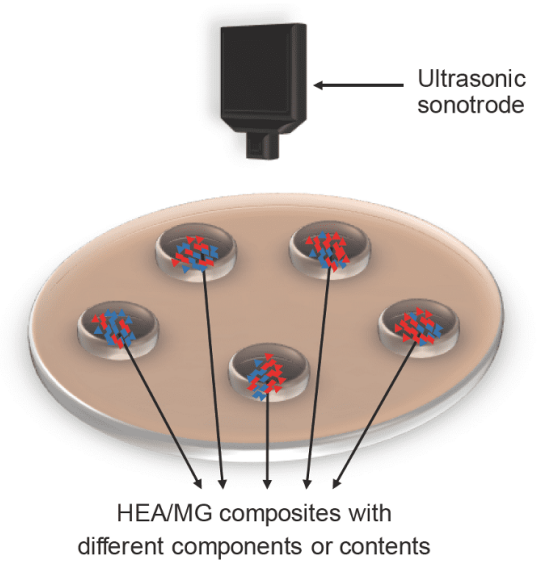

Figure 1 (Color online) Schematic for high-throughput preparation using ultrasonic hammering.

*Corresponding author (email: drzhangy@ustb.edu.cn) 
tios are adjustable. The components and contents of the feedstock materials can be tuned exactly according to specific requirements. As a result, it is conceivable that this technique would be quite useful in the compositional design and high-throughput preparation of amorphous composite, as shown in Figure 1, so as to further break through the limit of strength and ductility.

1 Li R, Wang Z, Guo Z, et al. Graded microstructures of Al-Li-Mg-Zn$\mathrm{Cu}$ entropic alloys under supergravity. Sci China Mater, 2019, 62:
736-744

2 Yan X H, Ma J, Zhang Y. High-throughput screening for biomedical applications in a Ti-Zr-Nb alloy system through masking co-sputtering. Sci China-Phys Mech Astron, 2019, 62: 996111

3 Luo F, Sun F, Li K, et al. Ultrasonic assisted micro-shear punching of amorphous alloy. Mater Res Lett, 2018, 6: 545-551

$4 \mathrm{Li} \mathrm{R}, \mathrm{Li} \mathrm{X}$, Ma J, et al. Sub-grain formation in Al-Li-Mg-Zn-Cu lightweight entropic alloy by ultrasonic hammering. Intermetallics, 2020, 121: 106780

5 Liang X, Zhu X L, Li X, et al. High-entropy alloy and amorphous alloy composites fabricated by ultrasonic vibrations. Sci China-Phys Mech Astron, 2020, 63: 116111 